\title{
Impact of symptoms by gender and age in Japanese subjects with irritable bowel syndrome with constipation (IBS-C): a large population-based internet survey
}

\author{
Masanori Kosako ${ }^{1 *} \mathbb{D}$, Hiraku Akiho ${ }^{2}$, Hiroto Miwa ${ }^{3}$, Motoyori Kanazawa ${ }^{4}$ and Shin Fukudo ${ }^{4}$
}

\begin{abstract}
Background: Irritable bowel syndrome with constipation (IBS-C) is a representative psychosomatic disorder. Several pathophysiological factors have been linked to IBS symptoms such as the modulation of gastrointestinal motility, visceral hypersensitivity, dysregulation of the gut-brain axis, genetic and environmental factors, sequelae of infection, and psychosocial disorders. It is likely that biopsychosocial aspects of IBS-C underlie its gender and age effects. However, the influence of each symptom of IBS-C by gender and age is not well understood. We hypothesized that the expression rate of each IBS-C symptom in females and in subjects aged 20-49 years was higher than that of subjects who were male and aged 50-79 years.
\end{abstract}

Methods: We conducted an internet survey of 30,000 adults from the general Japanese population. IBS-C subjects were asked to answer a questionnaire on the degree of anxiety, thoughts about bowel habits, and their dominant gastrointestinal symptoms together with exacerbation factors. The correlation between gender and age and IBS-C symptoms was analyzed.

Results: When analyzed by gender, the expression rate of abdominal discomfort, abdominal distention, and abdominal fullness was significantly higher in female than male IBS-C subjects $(66.5 \%$ vs. $58.7 \%, p<0.05 ; 54.7 \%$ vs. $43.6 \%, p<0.01$; $18.9 \%$ vs. $9.6 \%, p<0.01$, respectively). When analyzed by age, the expression rate of abdominal distention and abdominal pain was significantly higher among IBS-C subjects aged $20-49$ years than those aged $50-79$ years $(55.7 \%$ vs. $46.8 \%, p<0.05$; $36.6 \%$ vs. $20.6 \%, p<0.001$, respectively). In contrast, there was no gender or age differences with regard to the most common and bothersome symptom (abdominal bloating) among IBS-C subjects.

Conclusions: The expression rate of some IBS-C symptoms was higher among females and those aged 20-49 years than males and those aged 50-79 years, respectively. It is important to understand the impact of symptoms by gender and age to evaluate the pathology of IBS-C from a biopsychosocial perspective.

Trial registration: Although this survey was an anonymous internet survey, we obtained informed consent for the study as an online response. The disclosure of this study was approved by the Ethics Committee of Tohoku University Graduate School of Medicine (approval number: 2015-1-405).

Keywords: Irritable bowel syndrome (IBS), Constipation, Gender, Age, Abdominal bloating, Stress, Food, Epidemiology

\footnotetext{
* Correspondence: masanori.kosako@astellas.com

1Japan-Asia Clinical Development 1, Development, Astellas Pharma Inc.,

2-5-1, Nihonbashi-Honcho, Chuo-ku, Tokyo 103-8411, Japan

Full list of author information is available at the end of the article
}

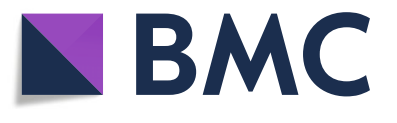

(c) The Author(s). 2018 Open Access This article is distributed under the terms of the Creative Commons Attribution 4.0 International License (http://creativecommons.org/licenses/by/4.0/), which permits unrestricted use, distribution, and reproduction in any medium, provided you give appropriate credit to the original author(s) and the source, provide a link to the Creative Commons license, and indicate if changes were made. The Creative Commons Public Domain Dedication waiver (http://creativecommons.org/publicdomain/zero/1.0/) applies to the data made available in this article, unless otherwise stated. 


\section{Background}

Irritable bowel syndrome (IBS) is a functional disorder that is characterized by persistent or recurrent gastrointestinal (GI) symptoms. IBS mainly causes abdominal pain or discomfort and bowel movement disturbance, but is not associated with an organic disease that can explain these symptoms [1]. According to the Rome III diagnostic criteria [1], which are international criteria for functional GI disorders, IBS is defined as "symptom onset at least 6 months prior to diagnosis, and recurrent abdominal pain or discomfort for $\geq 3$ days/month in the last 3 months. IBS is one kind of functional bowel disorder, associated with $\geq 2$ of the 3 items described in brackets [1. Improvement with defecation, 2 . Onset associated with a change in frequency of stool, 3. Onset associated with a change in form (appearance) of stool]". While IBS is not a fatal disease, the symptoms associated with IBS restrict patients' daily and social activities, and have been reported to significantly reduce patients' quality of life (QOL) [2, 3]. Based on the stool pattern at a particular point, IBS is classified into 4 subtypes: constipation (IBS-C), diarrhea (IBS-D), mixed (IBS-M), or unsubtyped IBS (IBS-U) [1]. Among these subtypes, IBS- $C$ is defined by hard or lumpy stools in $\geq 25 \%$ of bowel movements and loose (mushy) or watery stools in $<25 \%$ [1].

Several factors are thought to be related to IBS symptoms, including modulation of gastrointestinal motility, visceral hypersensitivity, dysregulated gut-brain axis, genetic and environmental factors, sequelae of infection, and psychosocial disorders. It is important to understand the disease from a biopsychosocial perspective.

Some reports have shown that the prevalence of IBS differs according to gender and age. Kanazawa et al. reported that approximately $16 \%$ of women and approximately $13 \%$ of men were diagnosed with IBS among survey subjects who received health check-ups [4]. A survey of the general Japanese population also found that the prevalence of IBS was significantly higher in women (1.7 times higher) than in men [5], which is consistent with data from other countries showing that the prevalence of IBS is generally higher in women. The prevalence of IBS is higher in individuals aged under 50 years than in those over 50 years [6]. IBS-C patients are often young and the percentage of female IBS-C patients is significantly higher than that of male IBS-C patients [7]. The higher prevalence of IBS in women compared with men may be associated with sex hormone fluctuations, which reportedly affect IBS symptoms, with symptoms appearing stronger before menstruation. Rectal susceptibility is also increased in women $[8,9]$. Generally, visceral perception decreases with age, and Wilms et al. reported an age-related decrease in abdominal pain perception [10]. Psychological and somatic symptoms in IBS may be associated with sex hormones and menstruation, as well as sex-related genes and social gender differences [11, 12], the gender-specific psychological way of illness perception, symptom awareness and coping with the disease. However, the prevalence of each symptom of IBS-C by gender and age is not well understood. We previously conducted survey of the current status of IBS and found that the prevalence of IBS-C according to the Rome III diagnostic criteria was $2.8 \%$ and that abdominal bloating was the most bothersome symptom [13]. However, we did not investigate the impact of gender or age on IBS-C symptoms. Given that Internet use decreases with increasing age, data obtained from a large number of elderly IBS-C subjects such as in this survey will be valuable.

Based on previous reports, we hypothesized that the expression rate of each IBS-C symptom was higher in females and those aged 20-49 years than in males and those aged 50-79 years, respectively. We verified this hypothesis by analyzing an internet survey to investigate the prevalence of IBS-C symptoms by gender and age.

\section{Methods}

This study used the same database as that published in our previous study [13] but conducted different hypotheses and analyses. While the aim of the previous study was to identify the most bothersome symptom in patients with IBS-C [13], the aim of the present study was to investigate the prevalence of IBS-C symptoms by gender and age. Details of the survey are described in the previous report [13]. Briefly, as a preliminary survey, 30,000 adults from the general Japanese population were recruited in October 28-31, 2013, for an internet survey to identify subtypes of IBS using a Macromill monitor panel (Macromill, Inc., Japan). Participants were asked to provide answers to questions regarding their living area, marriage status, children, income, and profession. Among the 30,000 participants, the screened subjects diagnosed with IBS-C using the Rome III criteria and the same number of age- and sex-matched non-IBS subjects randomly selected as controls were invited to participate in the main survey in November 1-4, 2013. In the main survey, IBS-C subjects were asked to answer a questionnaire on the degree of anxiety they experienced in their daily lives, the number of bowel movements they had in a week and thoughts about their bowel habits, and their dominant GI symptoms (including the most bothersome symptom) together with exacerbation factors, such as the circumstances and timing of symptoms and exacerbation. Degree of anxiety was assessed on a 4-point ordinate scale (0, Almost; 1 , Often; 2 , Sometimes; 3 , None) based on the Rome III diagnostic questionnaire. The details of the questionnaires were provided in our previous report as additional files [13].

The correlation between gender and age and IBS-C symptoms was secondarily analyzed after publication of our previous report [13]. Given that IBS symptoms 


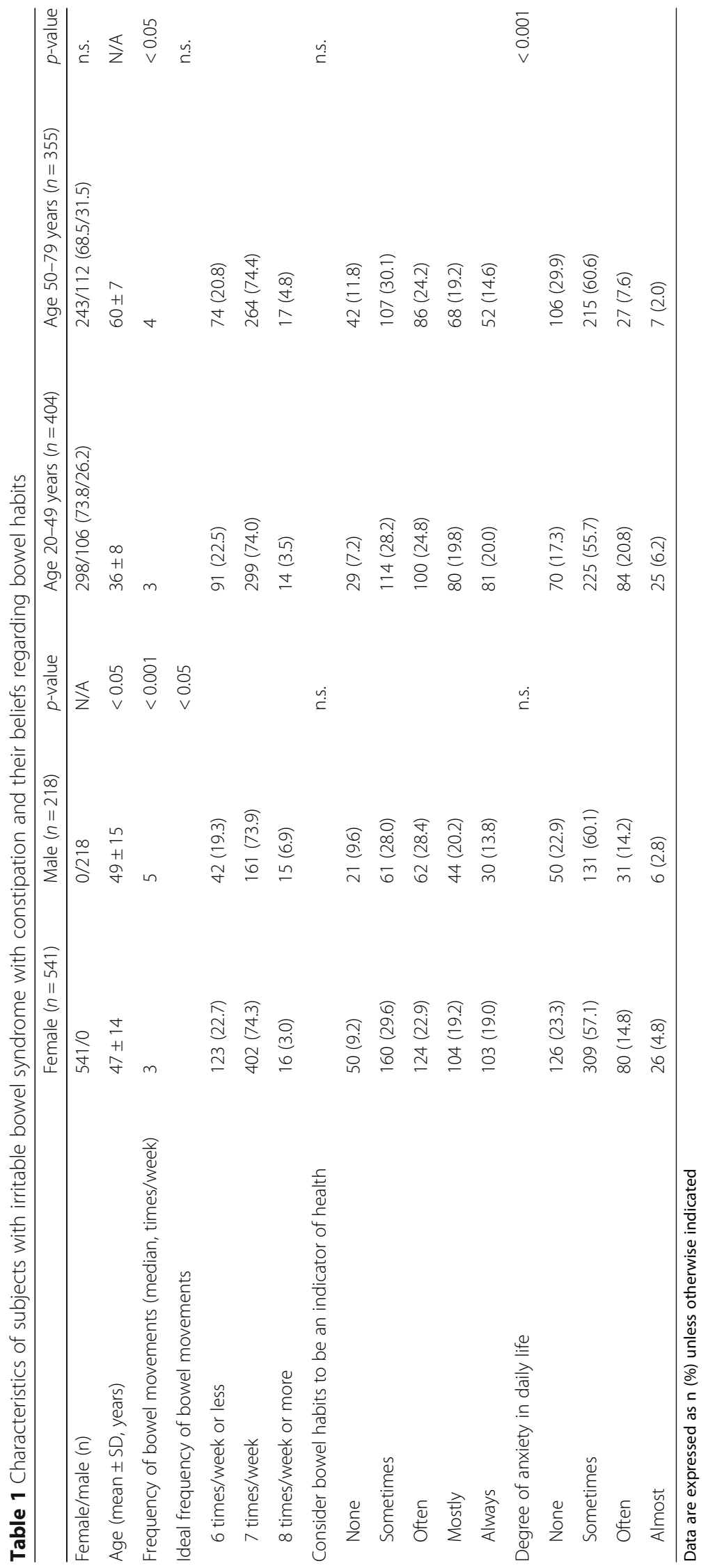


Table 2 Profession of IBS-C subjects

\begin{tabular}{lllll}
\hline Profession & Female $(\mathrm{n}=541)$ & Male $(\mathrm{n}=218)$ & Age 20-49 years $(\mathrm{n}=404)$ & Age 50-79 years $(\mathrm{n}=355)$ \\
\hline Civil servant & $7(1.3)$ & $9(4.1)$ & $7(1.7)$ & $9(2.5)$ \\
Manager and officer & $1(0.2)$ & $6(2.8)$ & $2(0.5)$ & $5(1.4)$ \\
Employee (administrative office) & $69(12.8)$ & $37(17.0)$ & $73(18.1)$ & $33(9.3)$ \\
Employee (engineering) & $6(1.1)$ & $31(14.2)$ & $25(6.2)$ & $12(3.4)$ \\
Employee (other) & $20(3.7)$ & $23(10.6)$ & $30(7.4)$ & $13(3.7)$ \\
Self-employed & $20(3.7)$ & $20(9.2)$ & $2(0.5)$ & $23(6.5)$ \\
Freelance & $5(0.9)$ & $6(2.8)$ & $106(26.2)$ & $9(2.5)$ \\
Homemaker & $229(42.3)$ & $2(0.9)$ & $74(18.3)$ & $50(14.1)$ \\
Temporary work & $103(19.0)$ & $21(9.6)$ & $22(5.4)$ & $0(0.0)$ \\
Student & $12(2.2)$ & $10(4.6)$ & $19(4.7)$ & $27(7.6)$ \\
Other & $27(5.0)$ & $19(8.7)$ & $27(6.7)$ & $49(13.8)$ \\
Not employed & $42(7.8)$ & $34(15.6)$ &
\end{tabular}

Data are expressed as $\mathrm{n}(\%)$ unless otherwise indicated

contains can vary, such as from GI symptoms to anxiety, we investigated differences in IBS symptoms between gender and age groups. Age was stratified into two groups, $<50$ years and $\geq 50$ years. Age $\geq 50$ years was selected as the threshold because it was close to the median age of the IBS-C subjects in this survey and is considered a risk factor by the Japanese guidelines for treatment of IBS [12]. Comparisons between two groups were conducted using the Mann-Whitney U-test or $\chi^{2}$ test. Associations between the symptoms and exacerbation factors among IBS-C subjects were evaluated using Kendall's $\mathrm{t}$-b. Analysis of multiplicity was not performed in the severity of the most bothersome symptom between two groups because each test was exploratory. Since the sample size of the age- and sex-matched subjects was low, analysis of age- and sex- matched subjects

Table 3 Association between the degree of anxiety and Gl symptoms in IBS-C subjects

\begin{tabular}{|c|c|c|c|c|c|c|c|c|c|c|}
\hline \multirow{2}{*}{$\begin{array}{l}\text { Gl symptoms/Degree } \\
\text { of anxiety }\end{array}$} & \multicolumn{5}{|c|}{ Female } & \multicolumn{5}{|l|}{ Male } \\
\hline & None & Sometimes & Often & Almost & Kendall's T & None & Sometimes & Often & Almost & Kendall's T \\
\hline Abdominal bloating & & & & & 0.08 & & & & & 0.09 \\
\hline No & 30 & 62 & 9 & 5 & & 12 & 29 & 4 & 0 & \\
\hline Yes & 96 & 247 & 71 & 21 & & 38 & 102 & 27 & 6 & \\
\hline Abdominal discomfort & & & & & $0.11^{*}$ & & & & & 0.04 \\
\hline No & 55 & 97 & 22 & 7 & & 21 & 56 & 12 & 1 & \\
\hline Yes & 71 & 212 & 58 & 19 & & 29 & 75 & 19 & 5 & \\
\hline Abdominal pain & & & & & $0.13^{*}$ & & & & & 0.06 \\
\hline No & 95 & 223 & 47 & 12 & & 37 & 100 & 23 & 1 & \\
\hline \multirow[t]{2}{*}{ Yes } & 31 & 86 & 33 & 14 & & 13 & 31 & 8 & 5 & \\
\hline & \multicolumn{5}{|c|}{ Age $20-49$ years } & \multicolumn{5}{|c|}{ Age $50-79$ years } \\
\hline Abdominal bloating & & & & & $0.12^{*}$ & & & & & 0.05 \\
\hline No & 19 & 52 & 9 & 4 & & 23 & 39 & 4 & 1 & \\
\hline Yes & 51 & 173 & 75 & 21 & & 83 & 176 & 23 & 6 & \\
\hline Abdominal discomfort & & & & & 0.09 & & & & & 0.08 \\
\hline No & 28 & 79 & 22 & 7 & & 48 & 74 & 12 & 1 & \\
\hline Yes & 42 & 146 & 62 & 18 & & 58 & 141 & 15 & 6 & \\
\hline Abdominal pain & & & & & $0.13^{*}$ & & & & & -0.01 \\
\hline No & 50 & 148 & 49 & 9 & & 82 & 175 & 21 & 4 & \\
\hline Yes & 20 & 77 & 35 & 16 & & 24 & 40 & 6 & 3 & \\
\hline
\end{tabular}

Gl gastrointestinal 
was not performed. The level of statistical significance was set at a $p$-value of less than 0.05 .

\section{Results \\ Gender}

Five hundred and forty-one female and 218 male IBS-C subjects completed the consecutive questionnaires in the main survey. The demographics of participants by gender are shown in Table 1 . The mean age \pm standard deviation (SD) of female IBS-C subjects was significantly lower than that of males $(47 \pm 14$ vs. $49 \pm 15, p<0.05)$. Female IBS-C subjects experienced lower frequency bowel movements $(p<0.001)$ and a lower ideal frequency of bowel movements $(p<0.05)$ than male IBS-C subjects. In contrast, there were no significant differences between female and male IBS-C subjects in whether participants considered bowel habit to be an indicator of health and in the degree of anxiety felt in daily life (Table 1). Professions of IBS-C subjects by gender are shown in Table 2.

The degree of anxiety was significantly associated with abdominal discomfort $(\tau=0.11, p<0.05)$ and abdominal pain $(\tau=0.13, p<0.05)$ but not abdominal bloating (Kendall's $\tau=0.08$, n.s.) in female IBS-C subjects. In contrast, the degree of anxiety was not significantly associated with abdominal bloating $(\tau=0.09$, n.s.) abdominal discomfort $(\tau=0.04$, n.s.), or abdominal pain ( $\tau$ $=0.06$, n.s.) in male IBS-C subjects (Table 3 ).

The expression rate of GI symptoms in female and male IBS-C subjects is shown in Fig. 1. Although abdominal bloating was the most common symptom associated with constipation in IBS-C subjects of both genders (female $80.4 \%$, male $79.4 \%$ ), the expression rate of almost all IBS symptoms was slightly higher among females than males. In particular, the expression rate of abdominal discomfort, abdominal distention, and abdominal fullness was significantly higher among female than male IBS-C subjects $(66.5 \%$ vs. $58.7 \%, p<0.05$; $54.7 \%$ vs. $43.6 \%, p<0.01 ; 18.9 \%$ vs. $9.6 \%, p<0.01$, respectively) (Fig. 1).

The expression rate of the most bothersome symptom in female and male IBS-C subjects is shown in Fig. 2. Abdominal bloating was reported as the most bothersome symptom in both female and male IBS-C subjects (female 30.1\%, male 21.1\%) (Fig. 2), and was most likely to occur after a meal (female 54.6\%, male 43.5\%) (Table 4). The most bothersome symptom (abdominal pain) in female and male subjects was most likely to occur during menstruation (48.0\%) and at work/school (44.4\%), respectively (Table 4 ). There was no significant

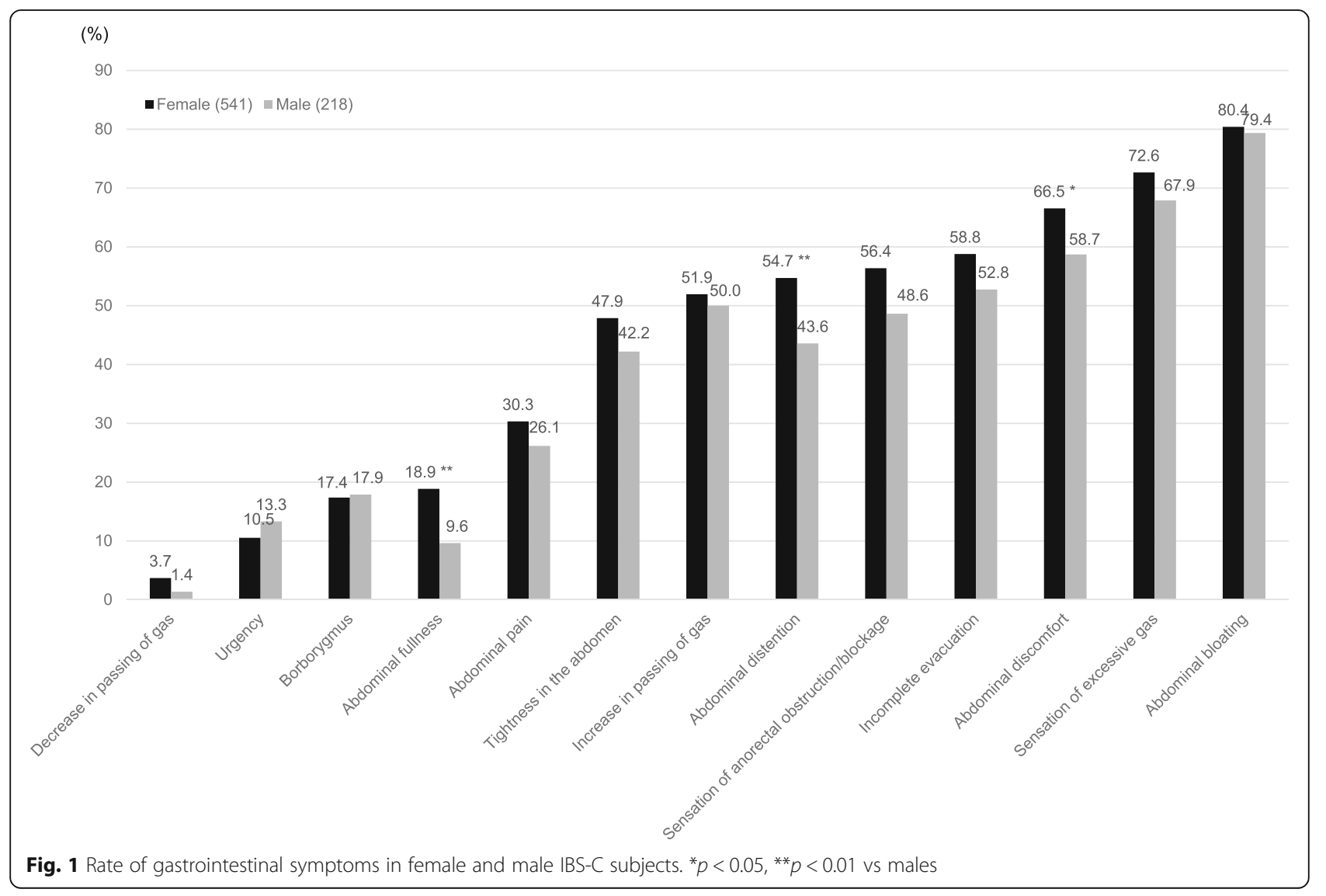




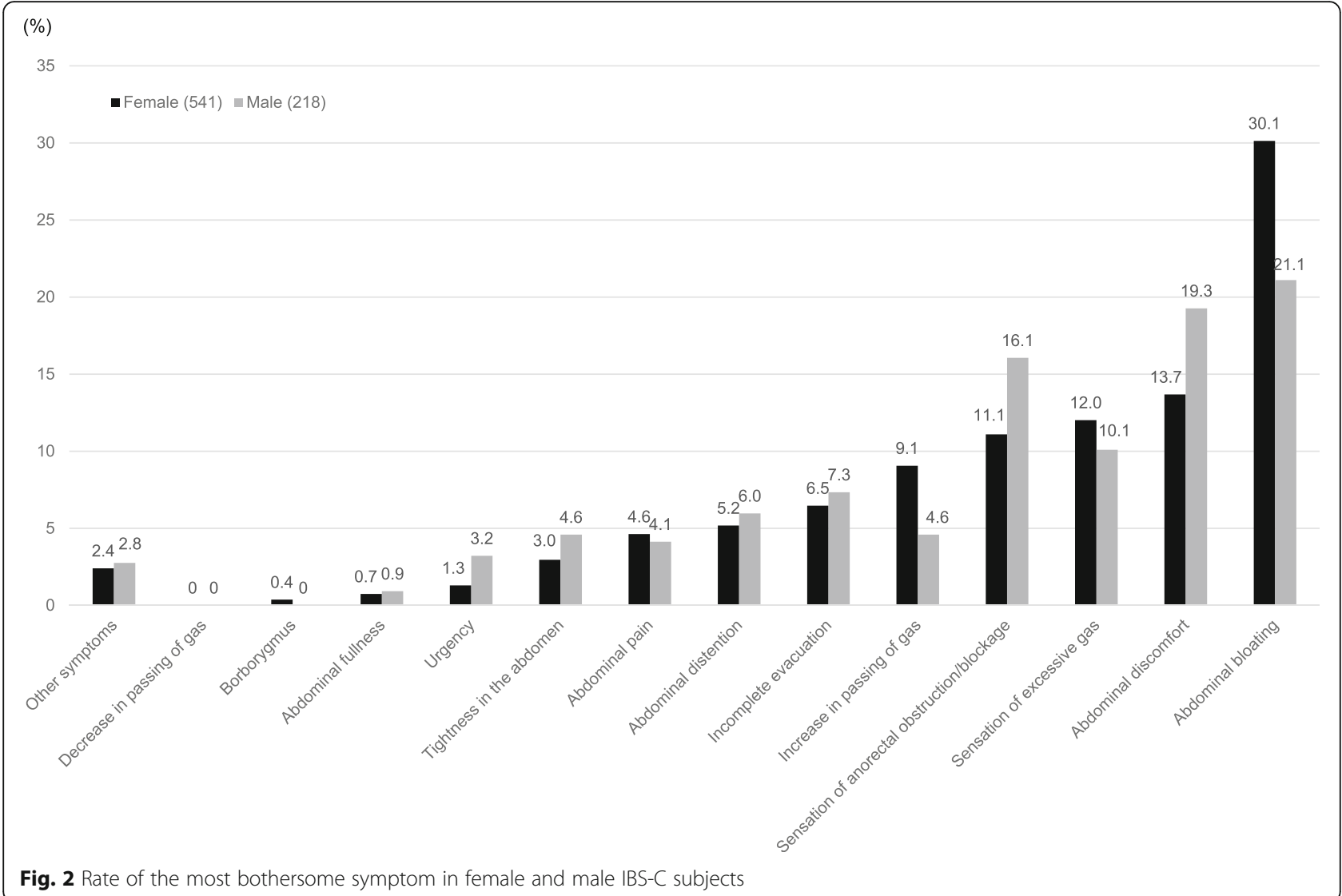

difference in the severity of the most bothersome symptom (abdominal bloating and abdominal pain) between female and male IBS-C subjects (Table 5). In contrast, the severity of the most bothersome symptom (abdominal discomfort) was significantly greater in female than male IBS-C subjects $(p<0.05)$.

\section{Age}

Four hundred and four IBS-C subjects aged 20-49 years and 355 aged 50-79 years completed the consecutive questionnaires. The demographics of the participants in each age group are shown in Table 1 . The mean age \pm SD of participants in the two age groups was $36 \pm 8$ and $60 \pm 7$ years. IBS-C subjects aged 20-49 years experienced lower frequency bowel movements $(p<0.05)$ and a higher degree of anxiety in their daily lives $(p<0.001)$ than those aged 50-79 years. In contrast, there was no significant difference between the two age groups in the ideal frequency of bowel movements, or participants' consideration of whether bowel habit was an indicator of health (Table 1). Professions of IBS-C subjects by age are shown in Table 2.

The degree of anxiety was significantly associated with abdominal bloating (Kendall's $\tau=0.12, p<0.05$ ) and abdominal pain $(\tau=0.13, p<0.05)$ but not abdominal discomfort $(\tau=0.09$, n.s. $)$ in IBS-C subjects aged 2049 years. In contrast, the degree of anxiety was not significantly associated with abdominal bloating $(\tau=0.05$, n.s.) abdominal discomfort ( $\mathrm{\tau}=0.08$, n.s.), or abdominal pain $(\tau=-0.01$, n.s.) in IBS-C subjects aged $50-79$ years (Table 3).

The expression rate of GI symptoms in IBS-C subjects aged 20-49 and 50-79 years is shown in Fig. 3. Although the most common symptom associated with constipation was abdominal bloating in both age groups (age $20-49$ years $79.2 \%$, age $50-79$ years $81.1 \%$ ), the expression rate of abdominal distention and abdominal pain was significantly higher among IBS-C subjects aged 20-49 years than those aged $50-79$ years $(55.7 \%$ vs. $46.8 \%, p<0.05$; $36.6 \%$ vs. $20.6 \%, p<0.001$, respectively) (Fig. 3).

The expression rate of the most bothersome symptom among IBS-C subjects aged $20-49$ and $50-79$ years is shown in Fig. 4. The most bothersome symptom among both age groups was abdominal bloating (age 2049 years $25.7 \%$, age $50-79$ years $29.6 \%$ ) (Fig. 4 ), and was most likely to occur after a meal (age $20-49$ years $51.9 \%$, age 50-79 years 52.4\%) (Table 4). Occurrence of the most bothersome symptom (abdominal pain) in IBS-C subjects aged $20-49$ and $50-79$ years was most likely to 


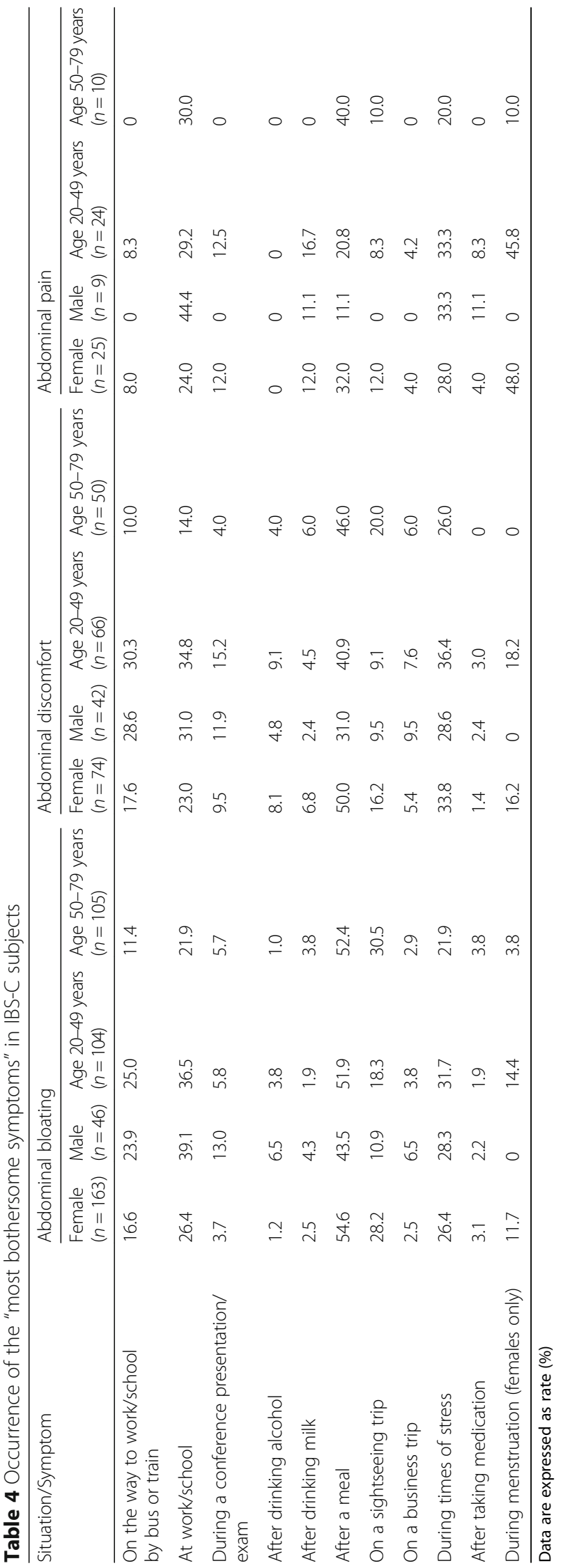


Table 5 Severity of the "most bothersome symptoms" in IBS-C subjects

\begin{tabular}{|c|c|c|c|c|c|c|c|}
\hline Gl symptom & 0 : Very mild & 1: Mild & 2: Moderate & 3: Severe & 4: Very Severe & Median & $p$-value \\
\hline Abdominal bloating & & & & & & & n.s \\
\hline Female $(n=163)$ & 0.6 & 4.3 & 31.9 & 46.6 & 16.6 & 3 & \\
\hline Male $(n=46)$ & 2.2 & 15.2 & 32.6 & 39.1 & 10.9 & 2.5 & \\
\hline Abdominal discomfort & & & & & & & $<0.05$ \\
\hline Female $(n=74)$ & 1.4 & 2.7 & 40.5 & 36.5 & 18.9 & 3 & \\
\hline Male $(n=42)$ & 0 & 16.7 & 54.8 & 19.0 & 9.5 & 2 & \\
\hline Abdominal pain & & & & & & & n.s. \\
\hline Female $(n=25)$ & 4.0 & 8.0 & 36.0 & 40.0 & 12.0 & 3 & \\
\hline Male $(n=9)$ & 0 & 11.1 & 22.2 & 55.6 & 11.1 & 3 & \\
\hline Abdominal bloating & & & & & & & n.s \\
\hline Age $20-49$ years $(n=104)$ & 1.0 & 7.7 & 33.7 & 40.4 & 17.3 & 3 & \\
\hline Age $50-79$ years $(n=105)$ & 1.0 & 5.7 & 30.5 & 49.5 & 13.3 & 3 & \\
\hline Abdominal discomfort & & & & & & & $<0.05$ \\
\hline Age $20-49$ years $(n=66)$ & 0 & 1.5 & 48.5 & 28.8 & 21.2 & 2.5 & \\
\hline Age $50-79$ years $(n=50)$ & 2.0 & 16.0 & 42.0 & 32.0 & 8.0 & 2 & \\
\hline Abdominal pain & & & & & & & n.s. \\
\hline Age $20-49$ years $(n=24)$ & 4.2 & 4.2 & 37.5 & 41.7 & 12.5 & 3 & \\
\hline Age $50-79$ years $(n=10)$ & 0 & 20.0 & 20.0 & 50.0 & 10.0 & 3 & \\
\hline
\end{tabular}

Gl gastrointestinal

Data are expressed as rate (\%)

occur during menstruation (45.8\%) and after a meal (40.0\%), respectively (Table 4 ). There was no significant difference in the severity of the most bothersome symptom (abdominal bloating and abdominal pain) between the two age groups (Table 5). In contrast, the severity of the most bothersome symptom (abdominal discomfort) was significantly greater among IBS-C subjects aged 2049 years than those aged $50-79$ years $(p<0.05)$.

\section{Discussion}

To our knowledge, this is the first study to report the expression of IBS-C symptoms in the Rome III by gender and age. We observed that the expression rate of abdominal discomfort, abdominal distention, and abdominal fullness was significantly higher among female than male IBS-C subjects. Moreover, the expression rate of abdominal distention and abdominal pain was significantly higher among IBS-C subjects aged 2049 than $50-79$ years. A study by Adeyemo et al. on overall IBS reported that female IBS subjects showed a greater prevalence of constipation-associated symptoms, particularly bloating and abdominal distension, than male IBS patients [11]. Studies reporting differences in the prevalence of symptoms between female and male IBS-C patients suggest that increased visceral sensitivity and sex hormones may be associated with the expression rate of IBS-C symptoms [7, 14, 15]. IBS-C symptoms may be associated with the gender-specific psychological way of illness perception, symptom awareness and coping with the disease. Visceral perception of abdominal pain in IBS-C patients may also decrease with age.

Our results showed that the most common symptom and the most bothersome symptom (abdominal bloating for both) did not differ by gender or age. Because we assumed that the Japanese term for "bloating" consisted of "abdominal bloating", "abdominal distention", "sensation of excessive gas", "tightness in the abdomen" and "abdominal fullness", we also surveyed the frequency of occurrence of these 5 specific symptoms in IBS-C subjects. Abdominal bloating is associated with decreased QOL and may lead to a higher frequency of physician visits [16]. Abdominal bloating is considered a key symptom among IBS patients in Asia [17], and may be an important reason prompting IBS-C patient consultations in Japan.

We previously reported that the most bothersome symptom (abdominal bloating) among IBS-C subjects was most likely to occur after a meal. This result did not change when subjects were stratified by gender and age. IBS symptoms such as abdominal pain and bloating occur or are exacerbated postprandially in approximately two-thirds of patients $[18,19]$. It is possible that the administration of IBS-C medications before meals will prevent the worsening of abdominal symptoms associated with anxiety. Young IBS-C subjects had a higher degree of anxiety in their daily lives than elderly subjects, while anxiety was similar between genders. In contrast, the 


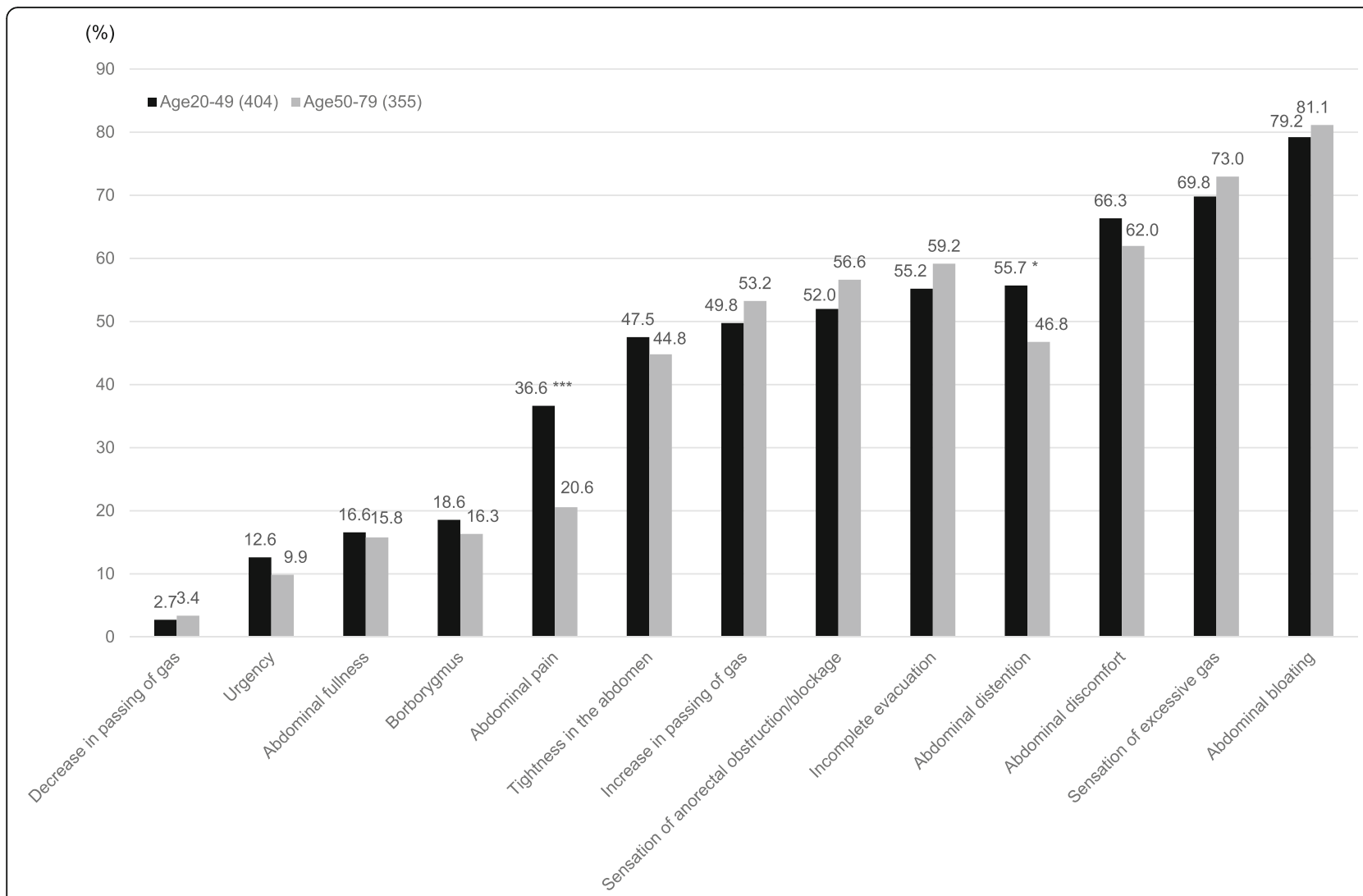

Fig. 3 Rate of gastrointestinal symptoms in IBS-C subjects aged $20-49$ and $50-79$ years. ${ }^{*} p<0.05,{ }^{* *} p<0.01,{ }^{* * *} p<0.001$ vs age $50-79$ years

degree of anxiety was correlated with some GI symptoms (abdominal bloating, abdominal discomfort, and abdominal pain) in young and female IBS-C subjects but not in elderly and male IBS-C subjects. Therefore, young IBS-C patients may be more suitable candidates for the biopsychosocial approach.

The most bothersome symptom (abdominal pain) in female IBS-C subjects and those aged 20-49 years occurred predominantly during menstruation. Houghton et al. reported that the menses in IBS is associated with a worsening of abdominal pain [9]. Together with our findings, this suggests that menses in female and young IBS-C subjects may be associated with a worsening of abdominal pain. In contrast, the most bothersome symptom (abdominal pain) in male IBS-C subjects occurred predominantly at work/ school and during times of stress. Although more female IBS-C subjects were homemakers compared with male IBS-C patients, male IBS-C subjects may feel more stress than female IBS-C subjects in their daily life. The severity of the most bothersome symptom (abdominal discomfort) in female and 20-49-year-old IBS-C subjects was significantly greater than that in male and 50-79-year old IBS-C subjects, respectively. Chang et al. reported that a number of gender-related factors may impact the clinical symptoms and response of IBS, such as gender roles, sociocultural differences, hormonal effects such as menstrual cycle variation, and biological differences influencing gut function and treatment response [20]. Suprathreshold distention of the rectum using a barostat induces abdominal discomfort and correspondingly causes higher activation of the medial prefrontal cortex and pregenual anterior cingulate cortex in IBS patients than in healthy controls [21]. Specifically, increasing age is associated with functional alterations in the blood-oxygen-level-dependent signal in the anterior cingulate cortex and medial prefrontal cortex [22]. It is likely that biopsychosocial aspects of IBS-C underlie its gender and age effects.

Several limitations of our study warrant mention. First, our study used an internet survey rather than a mail survey with random sampling from a list of residential areas in Japan. Subjects who are interested in their health may be more likely to participate in an internet survey. However, we collected a relatively large (30,000 participants) sample from a large monitor panel throughout Japan. Further, ascertainment bias is unlikely because previous reports indicate that the prevalence of overall IBS and IBS-C is similar to that of overall IBS and IBS-C using the Rome III diagnostic criteria in Japan $[7,23]$ and other countries [24]. Second, we could not exclude organic GI diseases and/or other comorbid diseases because we did not collect data on 


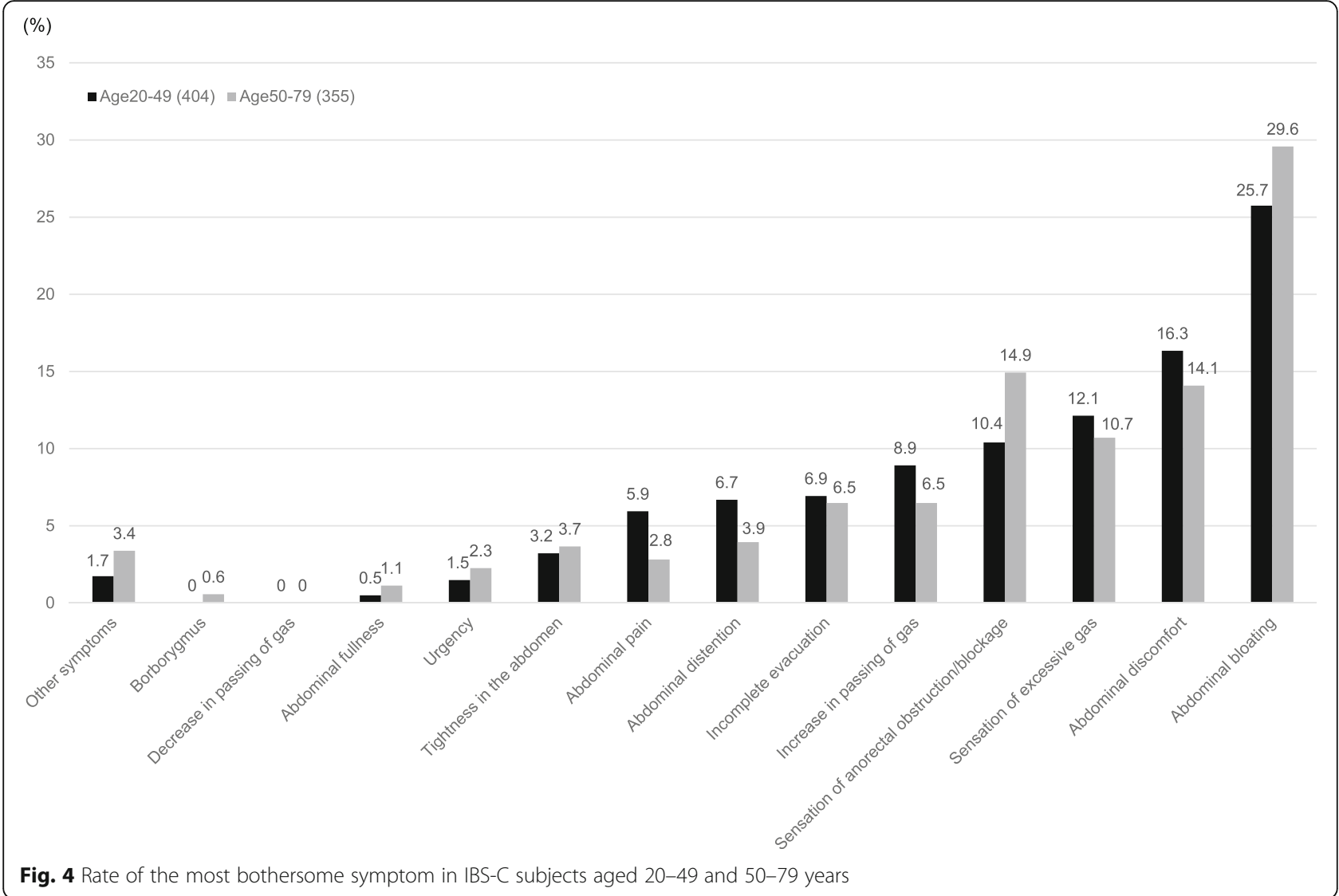

objective examination, prescription GI and psychiatric drugs, or those for other disease. IBS subjects have more somatic/psychiatric comorbidities that can affect their daily lives than non-IBS subjects [25]. However, the occurrence of abdominal discomfort or abdominal pain in our study population was similar to that in a previously reported population that excluded subjects with physician-diagnosed lower GI disorders [26], suggesting that organic GI diseases and/or other comorbid diseases likely had little effect on our findings. Moreover, our results did not include subjects aged $<20$ years, marital status, and education. The results for anxiety are limited by the use of a single item. Furthermore, the Rome III criteria were revised to Rome IV in May 2016, after this internet survey was conducted [27]. The main changes in the Rome IV criteria for IBS were the symptomatic frequency (Rome IV: $\geq 1$ day per week, Rome III: $\geq$ a few days per month) and the presence of abdominal pain (Rome IV: abdominal pain, Rome III: abdominal pain or discomfort). Future research based on the Rome IV criteria is warranted.

\section{Conclusion}

A large population-based internet survey suggests that the expression rate of some IBS-C symptoms is higher among female IBS-C subjects and those aged $20-49$ years than among males and those aged $50-79$ years, respectively. The degree of anxiety correlated with some GI symptoms (abdominal bloating, abdominal discomfort, and abdominal pain) in young and female IBS-C subjects but not in elderly and male IBS-C subjects. It is important to understand the impact of IBS-C symptoms by gender and age to evaluate the pathology of IBS-C from a biopsychosocial perspective.

\section{Abbreviations}

IBS: Irritable bowel syndrome; IBS-C: IBS with constipation; IBS-D: IBS with diarrhea; IBS-M: Mixed IBS; IBS-U: Unspecified IBS; GI: Gastrointestinal; QOL: Quality of life

\section{Acknowledgements}

The authors wish to thank Macromill, Inc. for supporting data aggregation. The authors would also like to thank Ayako Nakagawa for supporting data collection. Writing assistance was provided by DMC Corp.

\section{Funding}

This study was supported by a research grant from Astellas Pharma Inc., Tokyo, Japan.

Availability of data and materials

Datasets are available upon reasonable request.

\section{Authors' contributions}

MKosako is guarantor of this paper. HA, HM, SF and MKanazawa designed the study and critically reviewed the paper. MKosako performed the data 
analysis, interpretation of the results and drafting of the paper. All authors have approved the final manuscript.

\section{Ethics approval and consent to participate}

Although this survey was an anonymous internet survey, we obtained informed consent for the study as an online response. The disclosure of this study was approved by the Ethics Committee of Tohoku University School of Medicine (approval number: 2015-1-405).

\section{Consent for publication}

Not applicable.

\section{Competing interests}

The authors disclose the following: Masanori Kosako is an employee of Astellas Pharma, Inc.: Hiraku Akiho is a former employee of Astellas Pharma, Inc.: Hiroto Miwa and Shin Fukudo are recipients of research grants from Astellas Pharma, Inc. that are unrelated to this study; and Motoyori Kanazawa has no conflict of interest.

\section{Publisher's Note}

Springer Nature remains neutral with regard to jurisdictional claims in published maps and institutional affiliations.

\section{Author details}

1Japan-Asia Clinical Development 1, Development, Astellas Pharma Inc., 2-5-1, Nihonbashi-Honcho, Chuo-ku, Tokyo 103-8411, Japan. ${ }^{2}$ Former employee of Astellas Pharma Inc., Tokyo, Japan. ${ }^{3}$ Division of Gastroenterology, Department of Internal Medicine, Hyogo College of Medicine, Nishinomiya, Japan. ${ }^{4}$ Department of Behavioral Medicine, Tohoku University Graduate School of Medicine, Sendai, Japan.

Received: 21 May 2018 Accepted: 27 August 2018

\section{Published online: 04 September 2018}

\section{References}

1. Longstreth GF, Thompson WG, Chey WD, Houghton LA, Mearin F, Spiller RC. Functional bowel disorders. Gastroenterology. 2006:130:1480-91.

2. Gralnek IM, Hays RD, Kilbourne A, Naliboff B, Mayer EA. The impact of irritable bowel syndrome on health-related quality of life. Gastroenterology. 2000;119:654-60

3. Kaji M, Fujiwara $Y$, Shiba M, Kohata $Y$, Yamagami $H$, Tanigawa $T$, et al. Prevalence of overlaps between GERD, FD and IBS and impact on healthrelated quality of life. J Gastroen Hepatol. 2010;25:1151-6.

4. Kanazawa M, Endo $Y$, Whitehead WE, Kano M, Hongo M, Fukudo S. Patients and nonconsulters with irritable bowel syndrome reporting a parental history of bowel problems have more impaired psychological distress. Digest Dis Sci. 2004:49:1046-53.

5. Kumano H, Kaiya H, Yoshiuchi K, Yamanaka G, Sasaki T, Kuboki T. Comorbidity of irritable bowel syndrome, panic disorder, and agoraphobia in a Japanese representative sample. Am J Gastroenterol. 2004;99:370-6.

6. Fukudo $\mathrm{S}$, Kaneko $\mathrm{H}$, Akiho $\mathrm{H}$, Inamori $\mathrm{M}$, Endo $\mathrm{Y}$, Okumura $\mathrm{T}$, et al. Evidence-based clinical practice guidelines for irritable bowel syndrome. J Gastroenterol. 2015:50:11-30.

7. Kubo M, Fujiwara $Y$, Shiba M, Kohata $Y$, Yamagami $H$, Tanigawa $T$, et al Differences between risk factors among irritable bowel syndrome subtypes in Japanese adults. Neurogastroent Motil. 2011;23:249-54

8. Lee OY, Mayer EA, Schmulson M, Chang L, Naliboff B. Gender-related differences in IBS symptoms. Am J Gastroenterol. 2001;96:2184-93.

9. Houghton LA, Lea R, Jackson N, Whorwell PJ. The menstrual cycle affects rectal sensitivity in patients with irritable bowel syndrome but not healthy volunteers. Gut. 2002;50:471-4.

10. Wilms E, Jonkers D, Keszthelyi D, Mujagic Z, Vork L, Weerts ZZ, et al. Agerelated changes in abdominal pain in healthy individuals and Ibs patients. Gastroenterology. 2017:152:S647.

11. Adeyemo MA, Spiegel BMR, Chang L. Meta-analysis: do irritable bowel syndrome symptoms vary between men and women? Aliment Pharm Therap. 2010;32:738-55.

12. Gastroenterology JSo. The Guidelines for Treatment of IBS. 2014. http:// www.jsge.or.jp/files/uploads/IBSGL2_re.pdf.

13. Kanazawa M, Miwa H, Nakagawa A, Kosako M, Akiho H, Fukudo S. Abdominal bloating is the most bothersome symptom in irritable bowel syndrome with constipation (IBS-C): a large population-based internet survey in Japan. BioPsychoSocial Medicine. 2016:10:19.

14. Posserud I, Syrous A, Lindstrom L, Tack J, Abrahamsson H, Simren M. Altered rectal perception in irritable bowel syndrome is associated with symptom severity. Gastroenterology. 2007;133:1113-23.

15. Ouyang A, Wrzos HF. Contribution of gender to pathophysiology and clinical presentation of IBS: should management be different in women? Am J Gastroenterol. 2006;101:S602-S9.

16. Tuteja AK, Talley NJ, Joos SK, Tolman KG, Hickam DH. Abdominal bloating in employed adults: prevalence, risk factors, and association with other bowel disorders. Am J Gastroenterol. 2008:103:1241-8.

17. Gwee KA, Bak YT, Ghoshal UC, Gonlachanvit S, Lee OY, Fock KM, et al. Asian consensus on irritable bowel syndrome. J Gastroen Hepatol. 2010;25:1189-205.

18. Monsbakken KW, Vandvik PO, Farup PG. Perceived food intolerance in subjects with irritable bowel syndrome - etiology, prevalence and consequences. Eur J Clin Nutr. 2006;60:667-72.

19. Simren M, Mansson A, Langkilde AM, Svedlund J, Abrahamsson H, Bengtsson $U$, et al. Food-related gastrointestinal symptoms in the irritable bowel syndrome. Digestion. 2001;63:108-15.

20. Chang L, Toner BB, Fukudo S, Guthrie E, Locke GR, Norton NJ, et al. Gender, age, society, culture, and the patient's perspective in the functional gastrointestinal disorders. Gastroenterology. 2006;130:1435-46.

21. Kano M, Muratsubaki T, Van Oudenhove L, Morishita J, Yoshizawa M, Kohno $\mathrm{K}$, et al. Altered brain and gut responses to corticotropin-releasing hormone $(\mathrm{CRH})$ in patients with irritable bowel syndrome. Sci Rep. 2017;7:12425.

22. Rieck JR, Rodrigue KM, Boylan MA, Kennedy KM. Age-related reduction of BOLD modulation to cognitive difficulty predicts poorer task accuracy and poorer fluid reasoning ability. Neuroimage. 2017;147:262-71.

23. Miwa H. Prevalence of irritable bowel syndrome in Japan: internet survey using Rome III criteria. Patients Prefer Adherence. 2008;2:143-7.

24. Krogsgaard LR, Engsbro AL, Bytzer P. The epidemiology of irritable bowel syndrome in Denmark. A population-based survey in adults $<=50$ years of age. Scand J Gastroentero. 2013:48:523-9.

25. Whitehead WE, Palsson O, Jones KR. Systematic review of the comorbidity of irritable bowel syndrome with other disorders: what are the causes and implications? Gastroenterology. 2002;122:1140-56.

26. Palsson OS, Whitehead WE, van Tilburg MAL, Chang L, Chey W, Crowell MD et al. Development and validation of the Rome IV diagnostic questionnaire for adults. Gastroenterology. 2016;150:1481-91.

27. Lacy BE, Mearin F, Chang L, Chey WD, Lembo AJ, Simren M, et al. Bowe Disorders. Gastroenterology. 2016;150:1393-407.

\section{Ready to submit your research? Choose BMC and benefit from:}

- fast, convenient online submission

- thorough peer review by experienced researchers in your field

- rapid publication on acceptance

- support for research data, including large and complex data types

- gold Open Access which fosters wider collaboration and increased citations

- maximum visibility for your research: over $100 \mathrm{M}$ website views per year

At BMC, research is always in progress.

Learn more biomedcentral.com/submissions 